\title{
A drug is effective if better than a harmless control
}

\section{Valid trials can still be held, as with HIVNET 012, when ethics rules out a placebo group.}

Sir - In his Correspondence letter "HIV drug remains unproven without placebo trial” (Nature 434, 137; 2005), Valendar Turner asks on what basis it can be claimed that the single-dose nevirapine regimen is effective in reducing mother-to-infant HIV transmission.

He points out that the placebo arm was dropped in the HIVNET 012 trial (of which we were team members) and notes the high variability in reported historical HIV transmission rates.

But he ignores the valid comparison made by the HIVNET 012 trial — whose conclusions were recently accepted by a US Institute of Medicine panel - between nevirapine and another drug, zidovudine, which had itself been tested against a placebo in other studies starting at least four weeks before labour.

The HIVNET 012 trial in Uganda originally placed HIV-infected pregnant women and newborns in three groups, randomly selected, to receive either the nevirapine regimen (a single dose of nevirapine to the mother during labour and a single dose to the baby shortly after birth), or a very short course of zidovudine from the onset of labour through the first week of the baby's life, or a placebo.

As noted by Turner, the placebo arm of HIVNET 012 was dropped shortly after the study began, when a Thai study showed that a short course of zidovudine, given for the last four weeks of pregnancy and during labour, reduced transmission by $50 \%$ compared with placebo (N. Shaffer et al. Lancet 353, 773-780; 1999).

Women on HIVNET 012 continued to be enrolled in the two active drug arms, following recommendations - made jointly by the US Centers for Disease Control and Prevention, the National Institutes of Health, the French National Agency for AIDS Research, and the Joint United Nations Programme on HIV/AIDS — to drop the placebo arm from all ongoing perinatal HIV trials.

Once the placebo arm was dropped, the redesigned trial compared the mother-to-infant transmission rates for nevirapine and on zidovudine within the same trial, rather than making a comparison with historical transmission rates, as Turner implies.

When one compares two drugs, if the test and control drugs are found to be similar in efficacy, it is not possible to know whether the two drugs are equally effective or equally ineffective - unless there is clear evidence that the control drug is effective. This is why use of placebos is advocated in settings where the active control has not been established to have efficacy that is clinically and statistically significant.

But when an experimental drug is found to be superior to a control that itself is not harmful (thus replacing a placebo), the effectiveness of the experimental drug is thereby established.

In the HIVNET 012 trial, a very short course of zidovudine was used as the active control. Many studies had already reported that longer zidovudine regimens were more effective than placebos.

Of the HIVNET 012 patients who gave birth before discontinuation of the placebo arm, the transmission rate at 6-8 weeks of age was $37 \%$ in the placebo group, compared with $28 \%$ in the zidovudine group and $7 \%$ in the nevirapine group. (The 6-8-week transmission rates at the end of the trial were $20 \%$ for zidovudine and $11.8 \%$ for nevirapine.)

Most people would conclude that zidovudine does not increase the risk of transmission. Hence, our finding that single-dose nevirapine was significantly more effective in preventing HIV transmission than a very short course of zidovudine justifies the conclusion that the HIVNET 012 nevirapine regimen is more effective than nothing in preventing mother-to-infant HIV transmission.

Brooks Jackson ${ }^{\star}$, Thomas Fleming $\dagger$ ${ }^{\star}$ Johns Hopkins School of Medicine, 600 North Wolfe Street, Baltimore, Maryland 21287, USA $\dagger$ Department of Biostatistics,

University of Washington, Box 357232,

Seattle, Washington 98195, USA

\section{DNA barcoding does not compete with taxonomy}

Sir-M. C. Ebach and C. Holdrege, in Correspondence ("DNA barcoding is no substitute for taxonomy" Nature 434, 697; 2005), express some key misunderstandings regarding what a comprehensive DNA barcoding programme would - and especially would not - do. DNA barcoding projects are already achieving positive results, albeit on a relatively small scale. If implemented globally, DNA barcoding would benefit, not compromise, taxonomic science.

It is important to note that it does not seek to replace the linnaean system of classification, and thus differs fundamentally from proposals to create a new taxonomic system based solely on DNA. With only $15 \%$ of the estimated 10 million species described, large-scale DNA barcoding could be used to highlight probable new species within previously unstudied taxa. However, these species-in-waiting would not be named solely with a DNA barcode: they would be given linnaean names based on the study of curated voucher specimens, high-resolution digital images, collection locality data and other information.

Perhaps the most unfortunate misunderstanding is that DNA barcoding competes with taxonomy for funding. Existing DNA barcoding networks have been funded by agencies that do not have a tradition of supporting taxonomic work. A global DNA barcoding initiative would be a 'big science' programme, and as such would compete for priority with projects of similar scale from physics, medicine and genomics - not taxonomy. Moreover, the bulk of such funds would ultimately be directed toward the collection and curation of specimens, not DNA sequencing.

Rather than draining support from taxonomy, the DNA barcoding initiative has the potential to inject significant new funding into museums, herbaria and individual taxonomy labs.

\section{T. Ryan Gregory}

Department of Integrative Biology, University of Guelph, Guelph, Ontario N1G 2W1, Canada

\section{Nice planet, shame about the human race}

Sir — Inspired by Stephen Baxter's Futures story "Under martian ice" (Nature 433, 668 ; 2005), we began to discuss the Fermi paradox: that if aliens exist, they would have visited everywhere by now, including Earth. Careful consideration led us to conclude that if they were intelligent, they would not visit this planet.

Thus - and in opposition to the anthropic principle, which argues that the Universe is the way it is because we are here to observe it - we propose the misanthropic principle as the resolution of the puzzle.

Randall D. Kamien, Madhuri Kaul

Department of Physics and Astronomy, 209 South 33rd Street, University of Pennsylvania, Philadelphia 19104, USA

correspondence

Contributions to Correspondence may be submitted to corres@nature.com. 